$\left.y /\left(x^{2}+y^{2}\right)\right]=$ constant along $C$. That is, the inversion of the boundary with respect to the circle must also be a streamline of the original flow $f(z)$. To be generally admissible this inversion of the bqundary must be a streamline for every flow hence must itself be a rigid boundary. Thus a necessary and sufficient condition that rigid boundaries be generally admissible is that they be mapped into themselves under inversion with respect to the circle. A given boundary can be made conditionally admissible by obtaining the flow for this boundary together with its inversion and then regarding the inversion as only a streamline and not a boundary.

In particular, any plane barrier along $y=c x, a \leq x \leq b$, is generally admissible subject only to the condition that $a b=\left(1+c^{2}\right)^{-1}$. As an example of the application of the circle theorem under this modified restriction on rigid boundaries, consider

$$
f(z)=\frac{2}{3} \cos \theta[4 z-5]-i \frac{8}{3} \sin \theta\left[(z-2)\left(z-\frac{1}{2}\right)\right]^{1 / 2}
$$

representing flow at an angle $\theta$ about the plane boundary $1 / 2 \leq x \leq 2$. Then under the transformation $g(z)=f(z)+f^{*}(1 / z)$ it can be shown that both the unit circle and the plane boundary are streamlines. Any portion of this plane boundary is conditionally admissible with respect to this complex potential $f(z)$.

Acknowledgment. The author should like to express his gratitude to Professor R. Redheffer for helpful suggestions and criticism.

\title{
REFERENCES
}

1. L. M. Milne-Thomson, Theoretical hydrodynamics, 2nd ed., Macmillan and Co., London, 1949, p. 149.

2. L. M. Milne-Thomson, Hydrodynamical images, Proc. Camb. Phil. Soc. 36, 246-247 (1940).

\section{A NOTE ON A PAPER BY G. C. McVITTIE*}

\section{By G. B. WHITHAM (The University of Manchester)}

In a recent paper** in the Quarterly of Applied Mathematics G. C. McVittie uses Einstein's equations in general relativity to derive certain solutions of the classical equations of continuity and momentum for the compressible flow of a fluid when heat conduction and viscosity are neglected. Explicit expressions (which always satisfy these equations) are obtained for the density $\rho$, pressure $p$ and velocity components $U_{i}$ in terms of arbitrary functions $\phi, \phi_{1}, \phi_{2}, \phi_{3}$ of the time $T$ and space coordinates $X_{i}$; the $\phi$ 's are not independent however and must satisfy certain (differential) consistency equations. The equations of continuity and momentum are (using the double suffix summation convention)

$$
\begin{gathered}
\frac{\partial \rho}{\partial T}+\frac{\partial}{\partial X_{i}}\left(\rho U_{i}\right)=0 \\
\rho\left(\frac{\partial U_{i}}{\partial T}+U_{i} \frac{\partial U_{i}}{\partial X_{i}}\right)+\frac{\partial p}{\partial X_{i}}=0
\end{gathered}
$$

*Received December 2, 1953.

**G. C. McVittie, "A method of solution of the equations of classical gas dynamics, using Einstein's equations", Quart. of Appl. Math., 11, No. 3 (1953). 
McVittie's solutions are

$$
\begin{gathered}
U_{i}=-\frac{\partial^{2} \phi}{\partial X_{i} \partial T} / \nabla^{2} \phi \\
\rho=-\nabla^{2} \phi \\
p=-\frac{\partial^{2} \phi}{\partial T^{2}}+\frac{1}{3} \sum_{i=1}^{3}\left(\nabla^{2} \phi_{i}-\frac{\partial^{2} \phi_{i}}{\partial X_{i}^{2}}\right)+\frac{1}{3} \sum_{i=1}^{3}\left(\frac{\partial^{2} \phi}{\partial X_{i} \partial T}\right)^{2} / \nabla^{2} \phi
\end{gathered}
$$

where, if $l, m, n$ is a cyclic permutation of $1,2,3$,

$$
\frac{\partial^{2} \phi_{l}}{\partial X_{m} \partial X_{n}}=\frac{\partial^{2} \phi}{\partial X_{m} \partial T} \frac{\partial^{2} \phi}{\partial X_{n} \partial T} / \nabla^{2} \phi
$$

and

$$
\begin{aligned}
\frac{\partial^{2} \phi_{2}}{\partial X_{3}^{2}}+\frac{\partial^{2} \phi_{3}}{\partial X_{2}^{2}}+\left(\frac{\partial^{2} \phi}{\partial X_{1} \partial T}\right)^{2} \frac{1}{\nabla^{2} \phi} & =\frac{\partial^{2} \phi_{1}}{\partial X_{3}^{2}}+\frac{\partial^{2} \phi_{3}}{\partial X_{1}^{2}}+\left(\frac{\partial^{2} \phi}{\partial X_{2} \partial T}\right)^{2} \frac{1}{\nabla^{2} \bar{\phi}} \\
& =\frac{\partial^{2} \phi_{1}}{\partial X_{2}^{2}}+\frac{\partial^{2} \phi_{2}}{\partial X_{1}^{2}}+\left(\frac{\partial^{2} \phi}{\partial X_{3} \partial T}\right)^{2} \frac{1}{\nabla^{2} \bar{\phi}}
\end{aligned}
$$

In any problem $\phi_{1}, \phi_{2}, \phi_{3}$ must be eliminated from (6) and (7) so that $p, \rho, U_{i}$ are expressed in terms of $\phi$ alone; usually $\phi$ would then have to satisfy some energy relation.

The purpose of this note is to point out two results which would be of considerable value to anyone using this solution: (i) the elimination of $\phi_{1}, \phi_{2}, \phi_{3}$ can be carried out explicitly, and (ii) the results can be obtained in a few lines (and in fact more generally) directly from (1) and (2). This latter derivation is set out first. that

It is clear that (1) can be satisfied by the introduction of an arbitrary vector $A_{i}$ such

$$
\rho=\frac{\partial A_{i}}{\partial X_{i}}, \quad \rho U_{i}=-\frac{\partial A_{i}}{\partial T} .
$$

Equation (2) then expresses $\partial p / \partial X_{i}$ in terms of $A_{i}$. The simplest form is obtained if (1) is multiplied by $U_{i}$ and added to (2) to give

$$
-\frac{\partial p}{\partial X_{i}}=\frac{\partial}{\partial T}\left(\rho U_{i}\right)+\frac{\partial}{\partial X_{i}}\left(\rho U_{i} U_{i}\right)
$$

whence, on substitution from (8),

$$
-\frac{\partial p}{\partial X_{i}}=-\frac{\partial^{2} A_{i}}{\partial T^{2}}+\frac{\partial}{\partial X_{i}}\left\{\frac{\partial A_{i}}{\partial T} \frac{\partial A_{i}}{\partial T} / \frac{\partial A_{k}}{\partial X_{k}}\right\} .
$$

Equations (8) and (10) give explicit expressions for $\rho, U_{i}$ and $p$ in terms of the arbitrary vector $A_{i}$. A special case may be obtained by choosing $A_{i}=-\partial \phi / \partial X_{i}$; this assumes [from (8)] that curl $\rho U_{i}=0$, a condition which is always true if the flow depends only upon a single space variable and the time, but otherwise is restrictive. Then

$$
U_{i}=-\frac{\partial^{2} \phi}{\partial X_{i} \partial T} / \nabla^{2} \phi, \quad \rho=-\nabla^{2} \phi, \quad p=-\frac{\partial^{2} \phi}{\partial T^{2}}+\Phi,
$$


where $\Phi$ is determined in terms of $\phi$ by

$$
\frac{\partial \Phi}{\partial X_{i}}=\frac{\partial}{\partial X_{i}}\left\{\frac{\partial^{2} \phi}{\partial X_{i} \partial T} \frac{\partial^{2} \phi}{\partial X_{i} \partial T} / \nabla^{2} \phi\right\} .
$$

Expressions (11) and (12) are McVittie's results but with the dependence of $p$ on $\phi$ given explicitly. It is necessary to point out, however, that neither Eq. (10) nor Eq. (12) has a solution unless the right-hand side is an irrotational vector field. Since (12) is equivalent to McVittie's equations, this shows that these too have solutions only for a restricted class of $\phi$.

It is now shown how (3), (4), (j), (6), (7) may be reduced to (11) and (12). To eliminate $\phi_{1}, \phi_{2}, \phi_{3}$ in (5), (6), (7), an expression for the term $\frac{1}{3} \sum_{i=1}^{3}\left(\nabla^{2} \phi_{i}-\partial^{2} \phi_{i} / \partial X_{i}^{2}\right)$ in (5) is recuired in terms of $\phi$. However, if the three quantities which are equated in (7) are denoted by $E_{1}, E_{2}$ and $E_{3}$, respectively, and $\frac{1}{3}\left(E_{1}+E_{2}+E_{3}\right)$ is denoted by $\Psi$, it it seen that (5) is $p=-\partial^{2} \phi / \partial T^{2}+\Psi$; it remains, therefore, to demonstrate that $\Psi=\Phi$. Differentiating $E_{1}$ with respect to $X_{1}$ we have

$$
\frac{\partial \Psi}{\partial X_{1}}=\frac{\partial E_{1}}{\partial X_{1}}=\frac{\partial^{3} \phi_{2}}{\partial X_{3}^{2} \partial X_{1}}+\frac{\partial^{2} \phi_{3}}{\partial X_{2}^{2} \partial X_{1}}+\frac{\partial}{\partial X_{1}}\left\{\left(\frac{\partial^{2} \phi}{\partial X_{1} \partial T}\right)^{2} / \nabla^{2} \phi\right\} .
$$

Expressions for $\partial^{2} \phi_{2} / \partial X_{3} \partial X_{1}$ and $\partial^{2} \phi_{3} / \partial X_{2} \partial X_{1}$ are given in terms of $\phi$ by (6); hence $\partial \Psi / \partial X_{1}$ can be given in terms of $\phi$ alone. We have

$$
\begin{aligned}
\frac{\partial \Psi}{\partial X_{1}}= & \frac{\partial}{\partial X_{3}}\left\{\frac{\partial^{2} \phi}{\partial X_{3} \partial T} \cdot \frac{\partial^{2} \phi}{\partial X_{1} \partial T} / \nabla^{2} \phi\right\}+\frac{\partial}{\partial X_{2}}\left\{\frac{\partial^{2} \phi}{\partial X_{2} \partial T} \cdot \frac{\partial^{2} \phi}{\partial X_{1} \partial T} / \nabla^{2} \phi\right\} \\
& +\frac{\partial}{\partial X_{1}}\left\{\left(\frac{\partial^{2} \phi}{\partial X_{1} \partial T}\right) / \nabla^{2} \phi\right\} \\
= & \frac{\partial}{\partial X_{i}}\left\{\frac{\partial^{2} \phi}{\partial X_{j} \partial T} \cdot \frac{\partial^{2} \phi}{\partial X_{1} \partial T} / \nabla^{2} \phi\right\} .
\end{aligned}
$$

$\partial \Psi / \partial Y_{2}$ and $\partial \Psi / \partial X_{3}$ are obtained similarly and we observe that the expressions agree with (12); hence, $\Phi$ and $\Psi$ are indeed the same.

\section{BOOK REVIEWS}

An introduction to the theory of differential equations. By Walter Leighton. McGraw-Hill

Book Company, Inc., New York, Toronto, London, 1952. viii + 174 pp. $\$ 3.50$.

This book constitutes a carefully written exposition of the elements of the theory of ordinary linear differential equations. Accordingly, considerable attention has been given to questions of rigor, and to the understanding of basic concepts as opposed to mere formal facility of operation. To this end the author discusses the nature of solutions of differential equations and states and discusses existence theorems at appropriate points in the text. The proofs of these and other of the more difficult theorems are given, but in appendices for the sake of clarity of exposition.

The reviewer feels, however, that this book will be too difficult for the student who has just emerged from the first course in the calculus as given in most of our colleges. Frequent use is made of results ordinarily not met until a second calculus course is taken, although, where this is done, a statement to this effect is made or a reference given to material in the appendices. In several instances results are borrowed from more advanced differential equation theory, and in two cases reference is made to the theory of differential equations in the complex domain. This is presumably done for the purpose of presenting a 